\title{
JOURNAL OF ANIMAL SCIENCE
}

The Premier Journal and Leading Source of New Knowledge and Perspective in Animal Science

\section{Associations between osteochondrosis and conformation and locomotive characteristics in pigs}

D. B. de Koning, E. M. van Grevenhof, B. F. A. Laurenssen, B. J. Ducro, H. C. M. Heuven, P. N. de Groot, W. Hazeleger and B. Kemp

J ANIM SCI published online August 7, 2012

The online version of this article, along with updated information and services, is located on the World Wide Web at:

http://www.journalofanimalscience.org/content/early/2012/08/06/jas.2012-5310 
RUNNING HEAD: Osteochondrosis and conformation in pigs

Associations between osteochondrosis and conformation and locomotive characteristics in pigs ${ }^{1}$

D. B. de Koning* ${ }^{2,3}$, E. M. van Grevenhof $\dagger$, B. F. A. Laurenssen*, B. J. Ducro $\dagger$, H. C. M. Heuven $\dagger$, P. N. de Groot $\dagger$, and W. Hazeleger*, B. Kemp*

*Adaptation Physiology Group, Department of Animal Sciences, Wageningen University and Research centre, 6708 WD Wageningen, The Netherlands, †Animal Breeding and Genomics Centre, Department of Animal Sciences, Wageningen University and Research centre, 6708 WD Wageningen, The Netherlands, and $\ddagger$ Faculty of Veterinary Medicine, Utrecht University, 3508 TC Utrecht, The Netherlands

${ }^{1}$ The authors would like to express their gratitude to the personnel of the experimental accommodation 'De Haar' for their care of the animals and assistance throughout the experiment. This work was supported by the Science and Technology Foundation of the Netherlands Organization for Scientific Research (NWO-STW, grant number 11116), with cofinancers Institute for Pig Genetics BV (IPG BV), and Product Board Animal Feed (PDV).

${ }^{2}$ Corresponding author: danny.dekoning@wur.nl

${ }^{3}$ Current address: Adaptation Physiology Group, Department of Animal Sciences, Wageningen University and Research centre, 6708 WD Wageningen, The Netherlands 
ABSTRACT: Conformation and locomotive characteristics (CLC), i.e., leg conformation and gait movement patterns, may be associated with osteochondrosis (OC) in pigs. Osteochondrosis and CLC increase the risk of premature culling. This study investigated whether CLC have an explanatory value, over the previously modelled effects of sex, feeding, and housing conditions, on the occurrence and severity of OC in several joints and at the animal level. At 154 to $156 \mathrm{~d}$ of age, 267 pigs were subjectively scored on 9 conformation and 2 locomotive characteristics. Scoring was performed on a 9-point linear grading scale. For conformation characteristics, score 5 indicated normal conformation and scores 1 and 9 indicated severe deviations from normal. For the locomotive characteristics, score 1 indicated normal locomotion and score 9 indicated severe deviation from normal. At 161 to $176 \mathrm{~d}$ of age, pigs were slaughtered and joints were dissected for macroscopic evaluation of OC status. Results showed that swaying hindquarters and a stiffer gait were associated with higher scores for OC in, respectively, the femoropatellar ( $P=0.018)$ and tarsocrural joint $(P=0.005)$; smaller inner claws as compared to the outer claws of the front legs was associated with lower scores for OC than equally sized claws in the femoropatellar joint $(P=0.021)$ and on animal level $(P=0.010)$; steep and weak pasterns of the front legs were associated with higher scores for OC in the elbow joint $(P=0.004)$ and on animal level $(P=0.018)$; $\mathrm{X}$-shaped hind legs was associated with higher scores for OC on animal level $(P=0.037)$; and steep and weak pasterns of the hind legs were associated with lower scores for OC than normal conformation in the tarsocrural joint $(P=0.05)$. This study found several CLC that were associated with OC in several joints and at an animal level. This study showed that certain CLC might be used as indicators of OC and included in the criteria for selection of replacement animals for the breeding herd. 
Key words: conformation and locomotive characteristics, gait movement pattern, leg conformation, osteochondrosis, pig

\section{INTRODUCTION}

Conformation and locomotive characteristics (CLC), i.e. leg conformation and gait patterns, may be associated with osteochondrosis (OC) in pigs. OC in the epiphyseal growth plate involves a disturbed ossification process, which may cause deformation of the articular surface (reviewed by Ytrehus et al., 2007). As the articular surface is involved in joint movement, OC could have effects on CLC abnormalities (Jørgensen, 1995). On the other hand, CLC may serve as a causative factor for OC development. For example, biomechanical pressures within joints may be involved in OC development (Nakano and Aherne, 1988; Ytrehus et al., 2004a). Deviant pressures within joints may result from CLC abnormalities and in turn have an effect on OC.

Certain studies investigating OC show no signs of lameness in pigs (Carlson et al., 1986; Arnbjerg, 2007) or fail to show significant positive correlations (Jørgensen, 1995) with leg weakness (including CLC; Jørgensen et al., 1995). Other studies report positive associations between OC and leg weakness (Lundeheim, 1987; Stern et al., 1995; Jørgensen and Andersen, 2000), and increased culling probabilities with severity of leg weakness (Jørgensen and Sørensen, 1998; Jensen et al., 2010). However, differences in associations exist in strength and direction, and therefore the clinical relevance of OC is unclear.

If OC and CLC are associated, identification of OC affected pigs could be possible through CLC assessment, instead of assessment of joints after culling. These pigs could then be 
excluded from consideration for selection as breeding animals. Previously it was described how sex, feeding, and housing conditions affect OC status in pigs (van Grevenhof et al., 2011). The aim of this study is to investigate whether CLC have an explanatory value over sex, feeding, and housing conditions for OC status. This will provide insight in the use of in vivo measurements as an indicator of OC in pigs.

\section{MATERIALS AND METHODS}

\section{Ethical Note}

Osteochondrosis can cause joint surface irregularities possibly resulting in lameness of the animals that would endanger welfare of the pigs. Therefore, assessments for serious impairments of welfare in the pigs were performed daily. Severely lame or wounded pigs were taken out of the experiment and euthanized. All experiments were approved by the Animal Welfare Committee of Wageningen University and Research centre.

\section{Animals}

The experiment was performed in 2 batches. Batch 1 ( $\mathrm{n}=187 ; 82$ gilts, 105 intact boars) consisted of 'Tempo*Topigs 40’ crossbreds (Topigs, Vught, The Netherlands), descending from 23 dams. Batch 2 ( $\mathrm{n}=$ 157; 70 gilts, 87 intact boars) consisted of 'Pietrain*Topigs 40' crossbreds (Topigs, Vught, The Netherlands), descending from 18 dams. All pigs $(n=344)$ originated from an intermittent suckling experiment, which investigated the effects of different strategies for 
weaning piglets on sow reproductive performance and piglet performance (see for details Gerritsen et al., 2008; 2009). Briefly, piglets were either weaned conventionally at 3 wk of age, or with 12 continuous hours of separation from the sow a day (intermittent suckling) from d 14 or 21 after farrowing. Due to the intended effects of the intermittent suckling experiment, weaning age varied from $18.5 \mathrm{~d}$ to $49 \mathrm{~d}$ of age. Between weaning and the start of the experiment, the pigs were housed and fed in conventional fattening systems for these types of pigs. From farrowing to the start of treatments, water and feeding was available ad libitum. From weaning to slaughter, all animals had access to biting chains as enrichment.

\section{Treatments}

At 69 d of age, piglets were separated according to sex and per batch allocated to 32 pens of 5 to 6 individuals per pen (total of 64 pens over 2 batches). Mixing and grouping of the animals was performed based on an equal distribution of body weights measured at $42 \mathrm{~d}$ of age, the intermittent suckling treatment imposed, and litter mates. Pens (experimental units) were exposed to a $2 \times 2$ factorial treatment design of feeding level and housing type as described in van Grevenhof et al. (2011). Briefly, housing consisted of either $5 \mathrm{~m}^{2}$ of a $50 \%$ metal slatted (ridged round bars) and $50 \%$ solid concrete floor, or deep litter housing consisting of a concrete floor with 25- to 50-cm wood shavings as bedding. Feeding level was either ad libitum by an automatic feeding unit or restricted to $80 \%$ of the average daily ad libitum intake of the preceding week. Water was freely available in all pens through drinking nipples (for details see van Grevenhof et al., 2011). 


\section{CLC Assessment}

Due to research capacity, not all 344 pigs could be assessed. A random selection of pigs per batch and sex was therefore made for CLC assessment. Subjective scoring of CLC was performed in a total of 267 pigs (50 gilts and 63 boars from batch 1; 70 gilts and 84 boars from batch 2) between 154 and $156 \mathrm{~d}$ of age. Methodology was based on methods described by van Steenbergen (1989) and Jørgensen and Vestergaard (1990). Two locomotive and nine conformation characteristics were scored as described in Table 1 (see the aforementioned studies for figures describing the conformation characteristics). The two locomotive characteristics scored were swaying hindquarters and general gait movement / pattern. The scoring scale for the locomotive characteristics include a normal state at score 1, with scores above 1 indicating deviations in the locomotive characteristic (for example stiff gait or swaying hindquarters). One conformation characteristic was scored that does not involve leg conformation, which consisted of width of the hams. The front legs were scored for O shape or X shape, size of the claws, sickled or buckled shape, and steep or weak pasterns. The hind legs were scored for O shape or X shape, size of the claws, straight or sickled hock, and steep or weak pasterns. The scoring scale of all conformation characteristics contains a 'normal' leg conformation at score 5 and the severity in deviations above or below 5 indicates a certain type of characteristic (for example Xshaped legs or O-shaped legs). Scoring of the CLC for each pig was independently performed by two experienced judges unaware of the housing and feeding conditions, while pigs were individually walking freely in a concrete flooring hallway.

\section{OC Assessment}


At the end of the experiment, pigs were slaughtered at a local slaughterhouse to collect joints for macroscopical evaluation of irregularities of the articular surfaces, which are correlated to and indicative of OC (Jørgensen and Nielsen, 2005; Busch and Wachmann, 2010). Pigs were slaughtered in the range of 161 to $176 \mathrm{~d}$ of age with an average bodyweight of $102 \mathrm{~kg}$ (for details see van Grevenhof et al., 2012). After slaughter, carcasses were stored for $1 \mathrm{~d}$ at $4^{\circ} \mathrm{C}$ before collection of legs. Jørgensen and Andersen (2000) suggested that OC lesions show bilateral symmetry as genetic correlations of OC lesions in pigs between left and right legs approach 1. Therefore, only the left front and hind legs were collected from each pig through dissection of the shoulder and hip joints, respectively. These legs were then stored at $-21^{\circ} \mathrm{C}$ before assessment of joints.

The elbow, metacarpophalangeal, femoropatellar, tarsocrural, and metatarsophalangeal joints were dissected free from the legs to be macroscopically assessed for prevalence and severity of OC on a total of 24 locations (Table 2). Joints were scored on a grading scale from A to E (as described by van Weeren and Barneveld, 1999; van Grevenhof et al., 2011), with score A indicating no abnormalities and score E indicating severe abnormalities (for figures of the different classifications see van Grevenhof et al., 2011). OC was scored by a veterinarian, specialized in orthopaedics, unaware of the housing and feeding conditions, and experienced in judging OC.

\section{Statistics}


CLC data. The subjective scores of both judges were averaged and used as fixed covariates in statistical analyses. Weighted kappa coefficients and accompanying confidence intervals (CI) were calculated (Table 1) with PROC FREQ in SAS 9.2 (SAS Institute Inc. 2002 - 2008, Cary, NC, USA) using the default settings, as an indication of inter observer agreement (Landis and Koch, 1977).

For the conformation characteristics, deviations above or below score 5 relate to a certain characteristic (for example $\mathrm{X}$ - shaped legs or $\mathrm{O}$ - shaped legs), which both could result in differences of OC status. If a CLC is taken as a linear effect in statistical models, than it cannot account for scores below or above 5 both being associated with higher OC scores. Therefore, quadratic associations (using both the linear term and the accompanying quadratic term) of the conformation characteristics were assessed in statistical analyses to account for this type of scoring scale used. Additionally, because of this scale, the scores for the conformation characteristics need to be subtracted by the 'normal' score (score 5) for appropriate quadratic analyses. The reason for this is when mathematically the derivative of a quadratic relationship (y $=(x-5)^{2}+(x-5)+$ intercept; where $\mathrm{x}$ is a conformation score) is equated to 0 when the linear term is non-existent, it will result in the vertex of the parabola of the function at score $5(x=5)$. If no such transformation occurred $\left(y=x^{2}+x+\right.$ intercept), then the vertex of the parabola would result in score $0(\mathrm{x}=0)$ and would thus be incorrect considering the scale used. In this way, the statistical analyses will provide better insight of whether a linear term was existent. The locomotive characteristics are all on a linear scale with the optimum value at score 1 . Because no optimum value is present in the middle of the scoring scale, no quadratic relationships were assessed for the locomotive characteristics. 
Transformation of OC scores. For statistical analyses, the categorical OC observations (from A to E) were transformed to quantitative traits on a liability scale (for details see van Grevenhof et al., 2011). Briefly, the liability values were attained by superimposing an unobservable Gaussian response curve on the categorical OC observations. Dependent on the frequency distribution of the total categorical OC observations for one joint (joint locations of one joint summed over all animals), this resulted in a range of liability values that correspond to a phenotypic category for that joint. Consequently, each categorical OC observation for each location in one joint was transformed to the corresponding mean liability value calculated for that specific joint. This means that different liability values were calculated for each joint separately according to the locations and the categorical observations that occurred in a specific joint summed over all animals (the OC liability values will be referred to as OC scores). As described, OC scores were obtained from several locations within each joint. These OC scores per pig were summed for each joint individually to yield an overall OC score per joint (joint level). The total scores for each of the five joints per pig were then summed to yield an overall OC score (animal level).

Statistical models. To investigate associations of CLC on OC, the CLC were added as covariate fixed effects to the previously used model containing the fixed class effects of sex, feeding, and housing conditions (see van Grevenhof et al., 2011). As discussed before, quadratic relationships were assessed for the conformation characteristics and only linear relationships for the locomotive characteristics. A random component was added to the models which contained the random effects of dams from which the pigs descended. The genetic lines (one from each batch) did not show a significant influence (data not shown) on the analyses and were therefore not taken into any further account. Backward elimination was performed on the CLC with a stay-in 
$P$-value of $<0.1$ to attain the final statistical model. The fixed class effects at pen level and random effects remained in the models at all times to account for 'environmental' variation influencing OC status. This process was performed separately for the OC scores on the elbow joint, femoropatellar joint, tarsocrural joint, and for animal level, resulting in 4 statistical models. It was not performed for the metatarsophalangeal and metacarpophalangeal joints individually, because only 1 and 5 animals out of the 267 pigs assessed showed OC in the metatarsophalangeal and metacarpophalangeal joints, respectively. It was decided that statistical analyses for these two joints individually were inappropriate due to this very low number of animals showing OC in these two joints. However, the scores for these two joints were taken into account for the calculations on animal level (which is the total OC score of an animal for the elbow, metacarpophalangeal, femoropatellar, tarsocrural, and metatarsophalangeal joints). The general model used during backward elimination was as follows:

$Y_{i j k l m n o}=\mu+s_{i}+f_{j}+h_{k}+(f * h)_{j k}+p_{l(i j k)}+\left(\beta_{1} * C X+\beta_{2}{ }^{*} C X^{2}\right)_{m}+\left(\beta_{3} * l o\right)_{n}+d_{o}+e_{i j k l m n o}$

where

$Y_{i j k l m n o}=$ the $i j k l m n o^{\text {th }}$ observation of OC scores on joint level or animal level.

$\mu \quad=$ the mean.

$s_{i} \quad=$ the $i^{\text {th }}$ class effect of sex $(i=$ sow, boar $)$.

$f_{j} \quad=$ the $j^{\text {th }}$ class effect of feeding level $(j=$ ad libitum, restricted).

$h_{k} \quad=$ the $k^{\text {th }}$ class effect of housing system $(k=$ concrete partially slatted flooring, deep litter system).

$\left(f^{*} h\right)_{j k} \quad=$ the interaction effect of the $j^{\text {th }}$ feeding level and the $k^{\text {th }}$ housing system. 
$p_{l(i j k)}=$ the $l^{\text {th }}$ random level of the experimental unit pen nested within the

levels of $s_{i}, f_{j}$, and $h_{k}$, which was used as the error term for the specified fixed class effects.

$\left(\beta_{1}{ }^{*} c x+\beta_{2}{ }^{*} C X^{2}\right)_{m}=$ the regression coefficients $\beta_{1}$ and $\beta_{2}$ of the continuous linear $(c x)$ and quadratic $\left(c x^{2}\right)$ fixed terms for a certain conformation characteristic for which backward elimination was performed.

$\left(\beta_{3} * l o\right)_{n} \quad=$ the regression coefficients $\beta_{3}$ of the continuous linear fixed terms (lo) for a certain locomotive characteristic for which backward elimination was performed.

$d_{o} \quad=$ the random $o^{\text {th }}$ effect of dam $(o=\operatorname{dam} 1,2 \ldots .40,41)$.

$e_{i j k l m n o}=$ random residual term of the $i j k l m n o^{\text {th }}$ observation.

Influence statistics were performed to detect observations with a high leverage that possibly have a large effect or weight in a final model so that they predominantly determine the outcome of the analysis (Ott and Longnecker, 2001). Where appropriate, leverage issues will be presented.

The regression coefficients ( $\beta$ 's) associated with the covariates are on a liability scale. These results on a liability scale are difficult to interpret and to compare as they are calculated for each joint separately. Therefore, the regression coefficients and accompanying SE are presented in SD units. These are acquired by dividing regression coefficients $(\beta$ 's) and accompanying SE from one model by the SD of the response variable of that model (e.g. the SD of the OC scores of either the elbow joint, femoropatellar joint, tarsocrural joint, or animal level). All statistical analyses were performed using PROC MIXED in the statistical software package SAS 9.2 (SAS Institute Inc. 2002 - 2008, Cary, NC). 


\section{RESULTS}

Two out of the 267 pigs were euthanized before the end of the experiment due to health problems (data not shown). One animal was taken out due to severe lameness and the other animal was taken out due to severe tail-biting and an inflamed front leg.

\section{CLC Observer Agreement and Prevalence}

The CLC were evaluated by two judges and those scores were averaged per characteristic for statistical analyses. Agreement between the judges expressed in kappa coefficients (Table 1) ranged from 0.56 to 0.82 . The lowest agreements (kappa coefficient $<0.60$ ) were found for $\mathrm{O}$ shape - X shape of the hind legs and steep - weak pasterns of the hind legs. The highest agreement (kappa coefficient $>0.80$ ) was found for swaying hindquarters. Other CLC showed a range in kappa coefficients of $0.60-0.80$.

Prevalence of the CLC within the pigs assessed is shown in Table 3. Except for the locomotive characteristics (swaying hindquarters and gait / movement pattern) and width of the hams, the conformation characteristics of the pigs predominantly occurred in the scores 4 to 6 (69 \% or more of the pigs assessed in each characteristic) and fewer pigs occurred in the other scores. Less than $2 \%$ of the pigs assessed in each of the conformation characteristics occurred in the extreme scores (score 1 and 9).

\section{Associations between CLC and OC}


The aim of this study was to investigate whether CLC have an explanatory value over the effects of sex, feeding, and housing conditions on the prevalence and severity of OC in pigs. These effects of sex, feeding, and housing conditions on OC in these pigs have previously been modelled and described (van Grevenhof et al., 2011), and will not be presented here. Only the results relating to the explanatory value of the CLC on OC scores will be presented. For each of the results presented, the regression coefficients and $P$-values can be seen in Table 4. For only the significant results $(P \leq 0.05)$, the type of association is depicted in Figure 1 .

Elbow joint. Three conformation characteristics (steep - weak pasterns of the front legs, O shape - X shape of the hind legs, and straight - sickled hock) retained a $P$-value $<0.1$ in the statistical model for the elbow joint after backward elimination was applied. The significant quadratic association found between OC in the elbow joint and steep - weak pasterns of the front legs (Table 4) indicates that steep and weak pasterns were associated with higher OC scores in the elbow joint (Figure 1). The significant quadratic association found between OC in the elbow joint and O shape - X shape of the hind legs (Table 4) indicates that an O shaped or X shaped hind leg conformation was associated with lower OC scores in the elbow joint. However, only 2 and 4 pigs were scored as having $\mathrm{O}$ shaped or more severely $\mathrm{X}$ shaped hind legs, respectively (Table 3). Influence statistics (data not shown) revealed a high leverage for the two pigs with a conformation score 7 (both with a leverage of approximately 0.4 , which was approximately 10 times higher than the mean leverage). After omitting these two observations from the analysis, no association $(P>0.1)$ of $\mathrm{O}$ shape $-\mathrm{X}$ shape of the hind legs with OC scores in the elbow joint existed (no linear or quadratic association). The linear association found for straight - sickled 
hock (Table 4) shows that a sickled hock conformation tended to be associated with higher OC scores in the elbow joint than normal or straight hock conformations.

Femoropatellar joint. One locomotive characteristic (swaying hindquarters) and 2 conformation characteristics (claw size of the front legs and sickled - buckled front legs) retained a $P$-value $<$ 0.1 in the statistical model for the femoropatellar joint after backward elimination was applied. The significant linear association found for swaying hindquarters (Table 4) indicates that swaying hindquarters was associated with higher OC scores in the femoropatellar joint (Figure 1). The linear association found for claw size of the front legs (Table 4) indicates that smaller inner claws as compared to the outer claws was significantly associated with lower OC scores in the femoropatellar joint than equally sized claws (Figure 1). Only 1 pig was scored as having smaller outer claws (Table 3), indicating that the result for this conformation characteristic mainly pertains to smaller inner claws to equal claw size. The tendency for a quadratic association found for the conformation characteristic sickled - buckled front legs (Table 4) with OC in the femoropatellar joint indicates that a sickled or buckled front leg was associated with higher OC scores than a normal conformation. Influence statistics (data not shown) revealed that the only animal with a conformation score 9 for this characteristic in this analysis (data not shown) presented with a high leverage of approximately 0.36 (approximately 10 times higher than the mean leverage). After omitting this observation from the analysis, no association ( $P>$ 0.1) with OC scores in the femoropatellar joint was found for sickled - buckled front legs.

Tarsocrural joint. One locomotive characteristic (gait movement / pattern) and one conformation characteristic (steep - weak pasterns of the hind legs) retained a $P$-value $<0.1$ in 
the statistical model for the tarsocrural joint after backward elimination was applied. The linear association found for gait movement / pattern (Table 4) indicates that a slow and stiff gait pattern was significantly associated with higher OC scores in the tarsocrural joint than quicker and smoother gait patterns (Figure 1). The significant quadratic association found for steep - weak pasterns of the hind legs (Table 4) indicates that a steep or weak pastern was associated with lower OC scores than a normal conformation (Figure 1).

Animal level. One locomotive characteristic (swaying hindquarters) and 3 conformation characteristics (claw size of the front legs, steep - weak pasterns of the front legs, O shape $-\mathrm{X}$ shape of the hind legs) retained a $P$-value $<0.1$ in the statistical model for animal level after backward elimination was applied. The linear association found for swaying hindquarters (Table 4) indicates that swaying hindquarters tended to be associated with higher OC scores on animal level. Similar to the femoropatellar joint, smaller inner claws as compared to the outer claws of the front legs was significantly associated with lower OC scores on animal level (Table 4 and Figure 1). As before, 1 pig was scored as having smaller outer claws (Table 3), indicating that the result for this conformation characteristic mainly pertains to smaller inner claws to equal claw size. Similar to the elbow joint, the significant quadratic association found for steep - weak pasterns of the front legs (Table 4) indicates that a steep and weak pastern were associated with a higher OC score (Figure 1). A significant linear association on OC was found for the conformation characteristic O shape - X shape of the hind legs (Table 4) and indicates that an $\mathrm{X}$ shaped hind leg conformation was associated with higher OC scores on animal level than a normal leg conformation (Figure 1). The majority of animals occurred in the range of 
conformation score 5 to 6 (Table 3), indicating that the results mainly pertain to the normal to mild X shaped hind leg conformation. 


\section{DISCUSSION}

The aim of this study was to investigate whether CLC have an explanatory value on OC in several joints (elbow joint, femoropatellar joint, tarsocrural joint) and at the animal level, over the previously modelled and described effects of sex, feeding, and housing conditions (van Grevenhof et al., 2011). The CLC were subjectively scored on a 9-point grading scale by 2 evaluators. Several associations between CLC and OC were found and these may provide insight into the possibility of using CLC as in vivo measurements for OC status in pigs.

\section{CLC Observer Agreement}

The CLC were scored by 2 experienced observers on a 9-point grading scale. The scoring scale used allowed for the assessment of normal conformation or locomotion, or the degree of deviation from normal. Agreement between observers for the different CLC, expressed in kappa coefficients, ranged from 0.56 to 0.82 which indicates, respectively, “moderate agreement” to “almost perfect agreement” (Landis and Koch, 1977) between observers. The majority of the CLC scored showed a kappa coefficient range from 0.60 to 0.79 . These results suggest that there was "substantial agreement" between observers in scoring the animals similarly, but that there was still room for improvement in scoring some of the CLC. Disagreement between observations on the same animal is a known aspect in subjectively assessing different CLC in pigs (van Steenbergen, 1989), sheep (Janssens and Vandepitte, 2004; Janssens et al., 2004), horses (Mawdsley et al., 1996), and cattle (Veerkamp et al., 2002). In addition, some of these studies indicate only moderate repeatability of observations (agreement of scores for one animal 
assessed multiple times by the same observer) for different CLC. It is unlikely that complete agreement can be attained with subjectively scoring CLC. After all, normality and deviations from normality lie in the eye of the beholder and therefore are subject to one's own experience and views. However, it may be possible to reduce variation in agreements through training of observers and increased experience (Veerkamp et al., 2002; Janssens et al., 2004).

\section{CLC Prevalence}

In the majority of the pigs, many conformation characteristics occurred in the conformation scores between 4 and 6, while fewer pigs were scored in the other conformation scores. It may be possible that pigs with a more extreme leg conformation would show an even stronger significant relationship with occurrence and severity of OC (Lundeheim et al., 1987). However, other studies also report small variation in several CLC, with the majority of animals being classified as having relatively 'normal' leg conformation in pigs (van Steenbergen, 1989; Jørgensen and Vestergaard, 1990; Tarrés et al., 2006) and sheep (Janssens and Vandepitte, 2004; Janssens et al., 2004). Possibly, this small variation may have resulted from selection of animals in commercial husbandry against (severe) deviations from optimal states, as has been suggested for pigs (Tarrés et al., 2006) and horses (Mawdsley et al., 1996). For the locomotive characteristics, there was more variation in the number of pigs occurring in the different scores. This might indicate that variation in gait movement / pattern aberrations of pigs is more easily detectable by observers, which showed relatively high agreements for these characteristics.

The relatively small variation seen in CLC characteristics with few observations in the extreme CLC scores may have resulted in some of the leverage issues seen. If only a couple of 
animals are at the boundaries of the scoring scale while the majority is scored as (near) normal, then these couple of animals may have a large effect on statistical analyses (Ott and Longnecker, 2001). Therefore, influence statistics for such analyses has been performed to reveal these few observations with high leverage predominantly determining the outcome of an analysis. For example, a significant quadratic association between $\mathrm{O}$ shape $-\mathrm{X}$ shape of the hind legs and OC scores in the elbow joint was primarily caused by only 2 animals out of the 263 animals assessed. It therefore does not constitute as a reasonable association.

\section{CLC and $O C$}

In this study, several CLC were found to have a significant association with OC depending on the response variable assessed (joint level or animal level).

Elbow joint. Higher OC scores in the elbow joint were significantly associated with steep and weak pasterns of the front legs and tended to be associated with a sickled hock conformation. Other studies report various results for CLC to be associated with OC. Small to moderate associations of OC in the elbow joint were previously found with swaying hindquarters, stiff gait, buckled front legs, front or hind legs turned out (likely similar to X shaped leg conformation), upright or weak pasterns of the front legs, and sickled hock (Jørgensen, 1995; Jørgensen et al., 1995; Stern et al., 1995; Jørgensen, 2000; Jørgensen and Andersen, 2000; Luther et al., 2007; Kirk et al., 2008). However, these results are not always similar among studies (the type of conformation characteristics associated with OC, strength and the direction of association), and some dissimilarities may be caused by different breeds used among studies 
(van der Wal et al., 1987; Jørgensen and Andersen, 2000; and reviewed by Fukawa and Kusuhara, 2001). In the present study, 2 genetic lines were used (one for each batch), but no significant influence was shown when this aspect was taken into account in all analyses on joint level and animal level (data not shown) as was indicated also previously (van Grevenhof et al., 2011). Therefore, genetic lines were not taken into further account in the analyses.

Femoropatellar joint. Higher OC scores in the femoropatellar joint were significantly associated with a greater degree of swaying hindquarters. Lower OC scores were significantly associated with smaller inner claws as compared to the outer claws of the front legs. As lower OC scores were associated with smaller inner claws more than equally sized claws (at score 5), this might indicate that smaller inner claws 'protected' the femoropatellar joint from OC development. Small to moderate associations of OC in femoral condyles were found previously with swaying hindquarters, weak pasterns of the front legs, hind legs turned out (likely similar to X-shaped leg conformation), uneven claw size in the hind legs, weak or upright pasterns of the hind legs, and stiff gait (Stern et al., 1995; Jørgensen, 2000; Jørgensen and Andersen, 2000; Luther et al., 2007; Kirk et al., 2008). As with the elbow joint, results are dissimilar among studies and may indicate some breed differences (van der Wal et al., 1987; Jørgensen and Andersen, 2000; and reviewed by Fukawa and Kusuhara, 2001).

Tarsocrural joint. Higher OC scores in the tarsocrural joint were associated with a stiffer and slower gait movement / pattern, and lower OC scores were associated with steep and weak pasterns of the hind legs. For the steep - weak conformation, it seems that the optimum conformation is not at score 5, which was assumed in the scoring scale. Instead, the vertex of the 
quadratic relationship is found at score 4 (caused by the significant linear term), which is associated with a higher score for OC. The majority of animals also occurred in this conformation score 4 (136 out of 264 animals). As this score is assumed to indicate normality in the conformation, the deviations from score 4 and the lower OC scores associated with it, may indicate that steep or weak pasterns of the hind legs 'protected' the tarsocrural joint against OC development. Small to moderate associations of OC in the tarsocrural joint have previously been found, in (occasionally) different directions and magnitude between breeds, with weak or upright pasterns of the hind legs, stiff gait, and swaying hindquarters (Jørgensen and Andersen, 2000).

Animal level. Higher OC scores at the animal level tended to be associated with swaying hindquarters and were associated with steep - weak pasterns of the front legs, and X shaped hind leg conformation. Lower OC scores were associated with smaller inner claws as compared to the outer claws of the front legs. Considering that OC on animal level is a summation of all OC scores for the joints assessed, these CLC may suggest the more important CLC to monitor. This may be supported by the fact that at least 3of the 4 CLC also were found to have associations in individual joints. An association of swaying hindquarters and smaller inner claws as compared to the outer claws of the front legs with OC was also found in the femoropatellar joint, and steep weak pasterns of the front legs was also associated with OC in the elbow joint. The association between X-shaped hind leg conformation and OC may have had its effects within the tarsocrural joint and femoropatellar joint (main joints directly affected by this conformation). However, an association of X-shaped hind legs with OC was not found when the OC scores for both these joints were combined into 1 variable (data not shown). Considering that $\mathrm{O}$ shape $-\mathrm{X}$ shape of the hind legs had the lowest observer agreement, the majority of animals being scored as having 
normal leg conformation, and did not have an association with OC in individual joints, there may be some uncertainty for this result. Jørgensen and Andersen (2000) and Jørgensen (2000) suggested that the main components associated with OC are buckled front legs, legs turned out, upright (steep) pasterns in the hind legs, stiff locomotion, and swaying hindquarters, as confirmed by several associations found between OC on joint level or animal level in the present study. However, literature reports various (directions and magnitudes of) associations between OC in individual joints and certain conformation characteristics, whereas the locomotive characteristics seem to be more consistently associated with OC among studies (Jørgensen, 1995; Jørgensen et al., 1995; Stern et al., 1995; Jørgensen, 2000; Jørgensen and Andersen, 2000; Kirk et al., 2008). This may have something to do with most animals being scored as having a relatively normal conformation, whereas the locomotive characteristics seem to show more variation (as already discussed). The locomotive characteristic swaying hindquarters has been shown as a relatively stable characteristic through several parities, whereas this was not the case for several conformation characteristics (van Steenbergen et al., 1990). This might indicate that reproducibility in evaluation of conformation characteristics are variable depending on age, complicating how and to what extent conformation characteristics are associated with OC.

Implications. The analyses performed here only provide insight into the associations of CLC and OC. They do not provide insight into the causative factors to the occurrence of either OC or CLC. An increase in leg weakness, which includes CLC, might only be visible with an increase in severe OC (Jørgensen, 1995; Jørgensen et al., 1995). A pig with severe OC impairing its normal locomotive ability, might respond by redistributing its weight to alleviate this impairment resulting in a CLC problem. However, it might also be that an increase in severe OC lesions is 
caused by an increase in CLC abnormalities. Several studies have speculated that joint loading may be implicated in the development of OC (Nakano and Aherne, 1988; Carlson et al., 1991; Ytrehus et al., 2004a; Ytrehus et al., 2004b). Possibly, abnormal amount of pressure on certain (localized) parts of the joints may result from CLC abnormalities (Grøndalen and Grøndalen, 1974). This could lead to vascular disruption (Ytrehus et al., 2004a) or abnormalities in normal chondrocyte functioning (Quinn et al., 1999; Davisson et al., 2002) within the epiphyseal growth plate of joints, possibly resulting in OC. For example, the horizontal momentum generated when the hindquarters sway from left to right, may result in local overloading due to a constant redistribution of weight / pressures within the femoropatellar joint. Steep and weak pasterns of the front legs may cause local overloading on the posterior or anterior parts of the elbow joint. In addition, we found that there are associations between front leg conformation and OC scores in the hind legs (and vice versa), which has also been shown by Jørgensen et al. (1995) and Kirk et al. (2008). This suggests an even more complicated matter in which a certain leg conformation may influence weight distributions between front and hind legs. For example, a larger outer claw of the front legs may aid in the balance of an animal, as speculated for lateral digits in cattle (Muggli et al., 2011). Perhaps this may result in less correction necessary by the hind legs to remain in balance and less corrective pressures applied in the knee joint. Thus, effects of a certain leg conformation on OC may not be limited to only the legs in which the conformation is found. However, CLC can easily be assessed throughout a pigs life at several time points, whereas OC can usually only be assessed through radiographic monitoring (Jørgensen et al., 1995; Ebeid et al., 1998; Dik et al., 1999), only showing subchondral bone changes, or once after sacrificing the animal. Consequently, it is difficult to conclude whether OC lesions were present before or after the onset of CLC abnormalities. 
In conclusion, we found that swaying hindquarters, gait / movement pattern, steep - weak pasterns of the front and hind legs, and claw size of the front legs were significantly associated with the occurrence and severity of OC. These associations may serve as an early in vivo indicator of future problems in these pigs. A study by Dewey et al. (1993) showed that 34\% of pigs culled for lameness was associated with osteochondrosis and Yazdi et al. (2000) indicated reduced longevity with higher OC status. Proportions of animals culled due to locomotive problems or leg weakness are in the range of $8 \%$ to 31\% (Dagorn and Aumaitre, 1979; D'Allaire et al., 1987; Stein et al., 1990; Jørgensen and Sørensen, 1998; Scott et al., 2006; 2007). As OC and leg weakness are involved in the risk of premature culling, it might be possible to select these animals at risk for OC, with a certain CLC, at a young age and exclude them from breeding purposes. Especially since there are indications that CLC and OC have a genetic component (Lundeheim, 1987; Rothschild and Christian, 1988; Ytrehus et al., 2004b). Aside from the obvious welfare implications, one may speculate that this might lead to an increase of economic profit and durability in the commercial pig husbandry, as less animals will be prematurely culled at a later age or will produce offspring that are at an increased risk for development of CLC problems and OC or both.

\section{LITERATURE CITED}

Arnbjerg, J. 2007. Effect of a low-growth rate on the frequency of osteochondrosis in Danish Landrace pigs (short communication). Arch. Tierz. 50:105-111. 
Busch, M. E., and H. Wachmann. 2010. Osteochondrosis of the elbow joint in finishing pigs from three herds: Associations among different types of joint changes and between osteochondrosis and growth rate. Vet. J.: doi: 10.1016/j.tvjl.2010.1003.1021.

Carlson, C. S., H. D. Hilley, C. K. Henrikson, and D. J. Meuten. 1986. The ultrastructure of osteochondrosis of the articular-epiphyseal cartilage complex in growing swine. Calcif. Tissue. Int. 38:44-51.

Carlson, C. S., D. J. Meuten, and D. C. Richardson. 1991. Ischemic necrosis of cartilage in spontaneous and experimental lesions of ostochondrosis. J. Orthop. Res. 9:317-329.

D'Allaire, S., T. E. Stein, and A. D. Leman. 1987. Culling patterns in selected Minnesota swine breeding herds. Can. J. Vet. Res. 51:506-512.

Dagorn, J., and A. Aumaitre. 1979. Sow culling: Reasons for and effect on productivity. Livest. Prod. Sci. 6:167-177.

Davisson, T., S. Kunig, A. Chen, R. Sah, and A. Ratcliffe. 2002. Static and dynamic compression modulate matrix metabolism in tissue engineered cartilage. J. Orthop. Res. 20:842-848.

Dewey, C. E., R. M. Friendship, and M. R. Wilson. 1993. Clinical and postmortem examination of sows culled for lameness. Can. Vet. J. 34:555-556.

Dik, K. J., E. Enzerink, and P. R. van Weeren. 1999. Radiographic development of osteochondral abnormalities, in the hock and stifle of Dutch Warmblood foals, from age 1 to 11 months. Equine Vet. J. Suppl. 31:9-15.

Ebeid, M., W. Zimmerman, and G. Ueltschi. 1998. The radiographic diagnosis of osteochondrosis in pigs: A retrospective study. J. Swine. Health Prod. 6:121-125. 
Fukawa, K., and S. Kusuhara. 2001. The genetic and non-genetic aspects of leg weakness and osteochondrosis in pigs. Asian-Australas. J. Anim. Sci. 14:114-122.

Gerritsen, R., M. Berkeveld, N. M. Soede, P. Langendijk, M. A. M. Taverne, and B. Kemp. 2008. Feed intake and growth of piglets submitted to intermittent suckling regimens differing in timing and duration. Chapter 4 in Intermittent suckling and extended lactation. Improving adaptation of piglets to postweaning challenges. p 83-96. PhD diss. Faculty of Veterinary Medicine, Utrecht University, Utrecht.

Gerritsen, R., N. M. Soede, W. Hazeleger, P. Langendijk, S. J. Dieleman, M. A. M. Taverne, and B. Kemp. 2009. Intermittent suckling enables estrus and pregnanct during lactation in sows: Effects of stage of lactation and lactation during early pregnancy. Theriogenology 71:432-440.

Grøndalen, T., and J. Grøndalen. 1974. Osteochondrosis and arthrosis in pigs. IV. Effect of overloading on the distal epiphyseal plate of the ulna. Acta Agric. Scand. 15:53-60.

Janssens, S., and W. Vandepitte. 2004. Genetic parameters for body measurements and linear type traits in Belgian Bleu du Maine, Suffolk and Texel sheep. Small Rumin. Res. 54:1324.

Janssens, S., D. Winandy, A. Tylleman, C. Delmotte, W. van Moeseke, and W. Vandepitte. 2004. The linear assessment scheme for sheep in Belgium: breed averages and assessor quality. Small Rumin. Res. 51:85-95.

Jensen, T. B., M. K. Bonde, A. G. Kongsted, N. Toft, and J. T. Sørensen. 2010. The interrelationships between clinical signs and their effect on involuntary culling among pregnant sows in group-housing systems. Animal 11:1922-1928. 
Jørgensen, B. 1995. Effect of different energy and protein levels on leg weakness and osteochondrosis in pigs. Livest. Prod. Sci. 41:171-181.

Jørgensen, B. 2000. Osteochondrosis / osteoarthrosis and clawdisorders in sows, associated with leg weakness. Acta Vet. Scand. 41:123-138.

Jørgensen, B., and S. Andersen. 2000. Genetic parameters for osteochondrosis in Danish Landrace and Yorkshire boars and correlations with leg weakness and production traits. Anim. Sci. 71:427-434.

Jørgensen, B., J. Arnbjerg, and M. Aaslyng. 1995. Pathological and radiological investigations on osteochondrosis in pigs, associated with leg weakness. J. Vet. Med. A 42:489-504.

Jørgensen, B., and B. Nielsen. 2005. Genetic parameters for osteochondrosis traits in elbow joints of crossbred pigs and relationships with production traits. Anim. Sci. 81:319-324.

Jørgensen, B., and M. T. Sørensen. 1998. Different rearing intensities of gilts: II. Effects on subsequent leg weakness and longevity. Livest. Prod. Sci. 54:167-171.

Jørgensen, B., and T. Vestergaard. 1990. Genetics of leg weakness in boars at the Danish pig breeding stations. Acta Agric. Scand. 40:59-69.

Kirk, R. K., B. Jørgensen, and H. E. Jensen. 2008. The impact of elbow and knee joint lesions on abnormal gait and posture of sows. Acta Agric. Scand. 50:1-8.

Landis, J. R., and G. G. Koch. 1977. The measurement of observer agreement for categorical data. Biometrics 33:159-174.

Lundeheim, N. 1987. Genetic analysis of osteochondrosis and leg weakness in the Swedish pig progeny testing scheme. Acta Agric. Scand. 37:159-173. 
Luther, H., D. Schworer, and A. Hofer. 2007. Heritabilities of osteochondral lesions and genetic correlations with production and exterior traits in station-tested pigs. Animal 1:8:11051111.

Mawdsley, A., E. P. Kelly, F. H. Smith, and P. O. Brophy. 1996. Linear assessment of the thoroughbred horse: an approach to conformation evaluation. Equine Vet. J. 28:461-467.

Muggli, E., C. Sauter-Louis, U. Braun, and K. Nuss. 2011. Length asymmetry of the bovine digits. Vet. J. 188:295-300.

Nakano, T., and F. X. Aherne. 1988. Involvement of trauma in the pathogenesis of osteochondritis dissecans in swine. Can. J. Vet. Res. 52:154-155.

Ott, R. L., and M. Longnecker. 2001. Linear regression and correlation. Chapter 11 in An introduction to statistical methods and data analysis. Fifth edition. Duxbury, Pacific Grove, California, USA.

Quinn, T. M., A. A. Maung, A. J. Grodzinsky, E. B. Hunziker, and J. D. Sandy. 1999. Physical and biological regulation of proteoglycan turnover around chondrocytes in cartilage explants. Implications for tissue degradation and repair. Ann. N.Y. Acad. Sci. 878:420441.

Rothschild, M. F., and L. L. Christian. 1988. Genetic control of front-leg weakness in Duroc swine. I. Direct response to five generations of divergent selection. Livest. Prod. Sci. 19:459-471.

Scott, K., D. J. Chennells, D. Armstrong, L. Taylor, B. P. Gill, and S. A. Edwards. 2007. The welfare of finishing pigs under different housing and feeding systems: liquid versus dry feeding in fully-slatted and straw-based housing. Anim. Welf. 16:53-62. 
Scott, K., D. J. Chennells, F. M. Campbell, B. Hunt, D. Armstrong, L. Taylor, B. P. Gill, and S. A. Edwards. 2006. The welfare of finishing pigs in two contrasting housing systems: Fully-slatted versus straw-bedded accommodation. Livest. Sci. 103:104-115.

Stein, T. E., A. Dijkhuizen, S. D'Allaire, and R. S. Morris. 1990. Sow culling and mortality in commercial swine breeding herds. Prev. Vet. Med. 9:85-94.

Stern, S., N. Lundeheim, K. Johansson, and K. Andersson. 1995. Osteochondrosis and leg weakness in pigs selected for lean tissue growth rate. Livest. Prod. Sci. 44:45-52.

Tarrés, J., J. P. Bidanel, A. Hofer, and V. Ducrocq. 2006. Analysis of longevity and exterior traits on Large White sows in Switzerland. J. Anim. Sci. 84:2914-2924.

van der Wal, P. G., S. A. Goedegebuure, P. C. van der Valk, B. Engel, and G. van Essen. 1987. Leg weakness and osteochondrosis in pigs; Differences between the sexes of four breeds. Livest. Prod. Sci. 16:65-74.

van Grevenhof, E. M., H. C. M. Heuven, P. R. van Weeren, and P. Bijma. 2012. The relationship between growth and osteochondrosis in specific joints in pigs. Livest. Sci. 143:85-90. van Grevenhof, E. M., S. Ott, W. Hazeleger, P. R. van Weeren, P. Bijma, and B. Kemp. 2011. The effects of housing system and feeding level on the joint-specific prevalence of osteochondrosis in fattening pigs. Livest. Sci. 135:53-61.

van Steenbergen, E. J. 1989. Description and evaluation of a linear scoring system for exterior traits in pigs. Livest. Prod. Sci. 23:163-181.

van Steenbergen, E. J., E. Kanis, W. J. Koops, and H. A. M. van der Steen. 1990. Exterior in sows: 1 . Effect of parity number and association with reasons for disposal. Chapter 4 in Relevance of exterior appraisal in pig breeding. p 59-77. PhD diss. Department of Animal Breeding, Wageningen Agricultural University, Wageningen, The Netherlands. 
van Weeren, P. R., and A. Barneveld. 1999. The effect of exercise on the distribution and manifestation of osteochondrotic lesions in the Warmblood foal. Equine Vet. J. Suppl. $31: 16-25$.

Veerkamp, R. F., C. L. M. Gerritsen, E. P. C. Koenen, A. Hamoen, and G. de Jong. 2002. Evaluation of classifiers that score linear type traits and body condition score using common sires. J. Dairy Sci. 85:976-983.

Yazdi, M. H., N. Lundeheim, L. Rydhmer, E. Ringmar-Cederberg, and K. Johansson. 2000. Survival of Swedish Landrace and Yorkshire sows in relation to osteochondrosis: a genetic study. Anim. Sci. 71:1-9.

Ytrehus, B., C. S. Carlson, and S. Ekman. 2007. Etiology and Pathogenesis of steochondrosis. Vet. Pathol. 44:429-448.

Ytrehus, B., S. Ekman, C. S. Carlson, J. Teige, and F. P. Reinholt. 2004a. Focal changes in blood supply during normal epiphyseal growth are central in the pathogenesis of osteochondrosis in pigs. Bone 35:1294-1306.

Ytrehus, B., E. Grindflek, J. Teige, E. Stubsjøen, T. Grøndalen, C. S. Carlson, and S. Ekman. 2004b. The effect of parentage on the prevalence, severity and location of lesions of osteochondrosis in swine. J. Vet. Med. A 51:188-195. 
Table 1. Conformation and locomotive characteristics subjectively scored on a 9-point grading scale in pigs (modified from van Steenbergen [1989] and Jørgensen and Vestergaard [1990]) and interobserver agreement (kappa coefficient and accompanying 95\% confidence interval)

\begin{tabular}{lccc}
\hline Trait & \multicolumn{3}{c}{ Scoring } \\
\hline $\begin{array}{lccc}\text { Locomotive } \\
\text { characteristics }\end{array}$ & $\underline{\text { Score 1 }}$ & $\underline{\text { Score 9 }}$ & $\underline{\text { Kappa (95\% }}$ \\
Swaying hindquarters & No swaying & Severe & $0.82(0.77$ to \\
& Quick / & Swaying & $0.86)$ \\
Gait movement / pattern & smooth & Slow / difficult & $0.72(0.66$ to \\
& & & $0.78)$
\end{tabular}

\section{Conformation} characteristics $^{2}$

Width of hams

Front legs

O shape - X shape

Claw size ${ }^{3}$

Sickled - buckled

Steep - weak pasterns $\underline{\text { Score } 1 \text { Normal (5) }}$

Narrow $\underline{\text { Score } 9}$

Broad
Kappa (95\%

CI) ${ }^{4}$

$0.76(0.72$ to

$0.81)$

0.68 (0.60 to

Outer claw

$0.74(0.66$ to smaller smaller

Buckled

0.75 (0.68 to

Sickled

Steep

Weak

0.78 (0.72 to

\section{Hind legs}

O shape - X shape

O shape

X shape

$0.56(0.45$ to

Claw size ${ }^{3}$

Inner

claw smaller

Outer claw

$0.69(0.61$ to smaller

$0.76)$

Straight - sickled hock Straight

Sickled

$0.68(0.60$ to

$0.59(0.51$ to 0.67) 
${ }^{1}$ Locomotive characteristics were scored on a linear grading scale (1 to 9), where score 1 indicates normal locomotion and higher scores indicate a worse form of locomotion.

${ }^{2}$ Conformation characteristics were scored on a linear grading scale (1 to 9 ), where score 5 indicates 'normal' conformation and deviations from 5 indicate a different conformation. ${ }^{3}$ Claw size was also scored on a grading scale (1 to 9), but score 5 indicates equally sized claws instead of the classification normal.

${ }^{4}$ The kappa statistic is used as a measure of interobserver reliability from the 2 judges that evaluated the different characteristics on the same animals. A kappa of 0.01 to 0.20 is termed "slight agreement"; 0.21 to 0.40 is termed "fair agreement"; 0.41 to 0.60 is termed "moderate agreement"; 0.61 to 0.80 is termed "substantial agreement"; 0.81 to 0.99 is termed "almost perfect agreement” (Landis and Koch, 1977). 
Table 2. The locations within joints assessed for osteochondrosis

\begin{tabular}{ll}
\hline Joint & Joint location \\
\hline Elbow joint & Medial humeral condyle \\
& Lateral humeral condyle \\
& Anconeal process \\
& Proximal edge of radius \\
& Synovial fossa of radius \\
& Other locations \\
Metacarpophalangeal & Proximodorsal part of the \\
joint & sagittal ridge of the $3^{\text {rd }}$ \\
& metacarpophalangeal bone \\
Femoropatellar joint & Lateral femoral trochlea \\
& Medial femoral trochlea \\
joint & Sulcus distal femur \\
& Patella \\
& Lateral femoral condyle \\
& Medial femoral condyle \\
& Other locations \\
Tarsocrural joint & Sagittal ridge of distal tibia \\
& Lateral trochlea of talus \\
& Medial trochlea of talus \\
& Lateral malleolus of tibia \\
& Medial malleolus of tibia \\
& Base of talus \\
& Lateral tibial cochlea \\
& Medial tibial cochlea \\
& Other locations \\
& Proximodorsal part of the \\
& sagittal ridge of the $3^{\text {rd }}$ \\
& metatarsophalangeal bone \\
\hline
\end{tabular}


Table 3. Frequencies (number of animals [n] and percentage (\%) of total number of animals) of the different scores for conformation ${ }^{1}$ and locomotive ${ }^{2}$ characteristics (CLC) occurring in the pigs assessed 


\begin{tabular}{ccccccccccc}
\hline & \multicolumn{11}{c}{ CLC scores } \\
1 to & 2 to & 3 to & 4 to & 5 & 5.5 & 6.5 & 7.5 & 8.5 \\
1.5 & 2.5 & 3.5 & 4.5 & & to 6 & to 7 & to 8 & to 9 \\
\hline
\end{tabular}

Locomotive

characteristics

Swaying hindquarters

$\begin{array}{lllllccccc}\mathrm{n} & 62 & 23 & 9 & 12 & \begin{array}{c}10 \\ 5\end{array} & 40 & 12 & 3 & - \\ \% & 23.3 & 8.6 & 3.4 & 4.5 & \begin{array}{c}39 . \\ 5\end{array} & 15.0 & 4.5 & 1.1 & -\end{array}$

$\begin{array}{llllllllllll}\text { Gait movement / pattern } & \mathrm{n} & 59 & 103 & 52 & 31 & 6 & 11 & 3 & 1 & 1\end{array}$

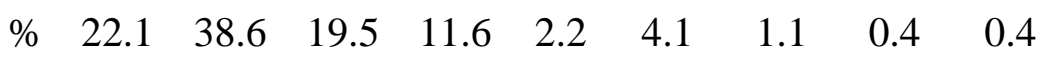

Conformation

characteristics

Width of hams

$\begin{array}{rrrrrrrrrr}\mathrm{n} & - & 4 & 20 & 36 & 52 & 68 & 49 & 34 & 3 \\ \% & - & 1.5 & 7.5 & 13.5 & \begin{array}{c}19 . \\ 5\end{array} & 25.6 & 18.4 & 12.8 & 1.1\end{array}$

Front legs

O shape $-X$ shape

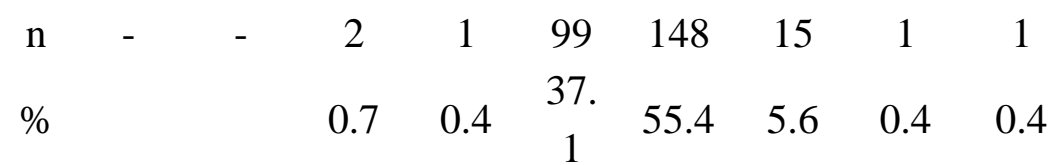

Claw size

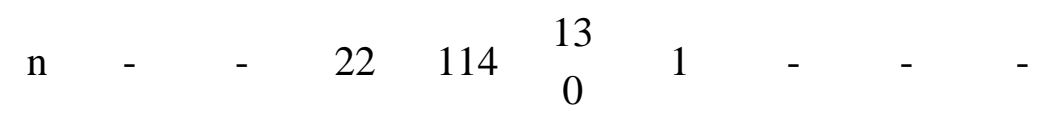

$$
\begin{array}{llllccc}
\% & - & - & 8.2 & 42.7 & 48 . & 0.4
\end{array}
$$

Sickled - buckled

$$
\mathrm{n} \quad-\quad \begin{array}{llllllll}
11 & & 1 & 4 & 116 & 21 & 6 & 2
\end{array}
$$$$
\begin{array}{llllllllll}
\% & - & - & 0.4 & 1.5 & \begin{array}{c}
43 . \\
6
\end{array} & 43.6 & 7.9 & 2.3 & 0.8
\end{array}
$$

$\begin{array}{llllllllllll}\text { Steep - weak pasterns } & \mathrm{n} & - & 4 & 12 & 39 & 71 & 75 & 49 & 13 & 3\end{array}$

$$
\begin{array}{llllllllll}
\% & - & \multicolumn{8}{c}{26 .} \\
& & 1.5 & 4.5 & 14.7 & 7 & 28.2 & 18.4 & 4.9 & 1.1
\end{array}
$$

Hind legs

O shape $-\mathrm{X}$ shape

$$
\begin{array}{cccccccccc}
\mathrm{n} & - & - & - & 2 & 18 & 76 & 4 & - & - \\
& & & & & 4 & & & & \\
\% & - & - & - & 0.8 & 69 . & 28.6 & 1.5 & - & -
\end{array}
$$

Claw size

$$
\mathrm{n} \quad-\quad \begin{array}{lllllll}
10 & 29 & 134 & 1 & 1 & -
\end{array}
$$




\begin{tabular}{cccccccccccccc} 
& & $\%$ & - & 0.7 & 10.9 & 50.2 & 37. & 0.4 & - & - & - \\
Straight - sickled hock & & & & & & 8 & & & & \\
& $\mathrm{n}$ & - & 1 & 10 & 74 & 13 & 37 & 8 & 3 & - \\
Steep - weak pasterns & $\%$ & - & 0.4 & 3.8 & 27.8 & 50 & 13.9 & 3.0 & 1.1 & - \\
& $\mathrm{n}$ & 4 & 12 & 41 & 136 & 63 & 9 & 1 & - & - \\
& $\%$ & 1.5 & 4.5 & 15.4 & 51.1 & 7 & 3.4 & 0.4 & - & - \\
\hline
\end{tabular}

${ }^{1}$ The locomotive characteristics were subjectively scored on a scale of 1 to 9 with 1 indicating normal locomotion and higher scores indicating a worse form of locomotion.

2 The conformation characteristics were subjectively scored on a scale of 1 to 9 . Score 5 indicates a normal leg conformation (deviations below or above 5 correspond, respectively, to the conformation characteristic in the name 'conformation name A conformation name B'), except for the claw size where 5 indicates equally sized claws (deviations below 5 relate to smaller inner claws, deviations above 5 relate to smaller outer claws). 
Table 4. Regression coefficients ( \pm SE) in SD units of conformation and locomotive characteristics associated with osteoc (OC) scores in the elbow joint, femoropatellar joint, tarsocrural joint, and on animal level ${ }^{1}$

\begin{tabular}{|c|c|c|c|c|c|c|c|c|}
\hline & \multicolumn{2}{|c|}{$\begin{array}{l}\text { Elbow joint } \\
(\mathrm{n}=263)\end{array}$} & \multicolumn{2}{|c|}{$\begin{array}{l}\text { Femoropatellar joint } \\
\qquad(\mathrm{n}=264)\end{array}$} & \multicolumn{2}{|c|}{$\begin{array}{l}\text { Tarsocrural joint } \\
(\mathrm{n}=264)\end{array}$} & \multicolumn{2}{|c|}{$\begin{array}{c}\text { Animal level } \\
(\mathrm{n}=259)\end{array}$} \\
\hline & Estimate & $\begin{array}{c}P- \\
\text { value }\end{array}$ & Estimate & $\begin{array}{c}P- \\
\text { value }\end{array}$ & Estimate & $\begin{array}{c}P- \\
\text { value }\end{array}$ & Estimate & $\begin{array}{c}P- \\
\text { value }\end{array}$ \\
\hline \multicolumn{9}{|l|}{ Locomotive } \\
\hline \multicolumn{9}{|l|}{ characteristics $^{2}$} \\
\hline Swaying hindquarters & & & $0.086 \pm 0.036$ & 0.018 & & & $\begin{array}{c}0.057 \pm \\
0.032\end{array}$ & 0.078 \\
\hline Gait movement pattern & & & & & $\begin{array}{c}0.149 \pm \\
0.052\end{array}$ & 0.005 & & \\
\hline \multicolumn{9}{|l|}{ Conformation } \\
\hline \multicolumn{9}{|l|}{ characteristics $^{3}$} \\
\hline \multicolumn{9}{|l|}{ Front legs } \\
\hline Claw size & & & $0.271 \pm 0.116$ & 0.021 & & & $\begin{array}{c}0.298 \pm \\
0.115\end{array}$ & 0.010 \\
\hline Sickled - buckled & & & $\begin{array}{c}-0.125 \pm \\
0.156\end{array}$ & $0.425^{4}$ & & & & \\
\hline Sickled - buckled quad & & & $0.116 \pm 0.063$ & $0.068^{4}$ & & & & \\
\hline Steep - weak pasterns & $\begin{array}{c}-0.065 \pm \\
0.060\end{array}$ & 0.278 & & & & & $\begin{array}{c}-0.076 \pm \\
0.061\end{array}$ & 0.215 \\
\hline Steep - weak pasterns quad & $\begin{array}{c}0.076 \pm \\
0.026\end{array}$ & 0.004 & & & & & $\begin{array}{c}0.061 \pm \\
0.026\end{array}$ & 0.018 \\
\hline \multicolumn{9}{|l|}{ Hind legs } \\
\hline O shape - X shape & $\begin{array}{c}0.793 \pm \\
0.345\end{array}$ & $0.023^{4}$ & & & & & $\begin{array}{c}0.304 \pm \\
0.145\end{array}$ & 0.037 \\
\hline O shape - X shape quad & $\begin{array}{c}-0.736 \pm \\
0.283\end{array}$ & $0.010^{4}$ & & & & & & \\
\hline Straight - sickled hock & $\begin{array}{c}0.140 \pm \\
0.079\end{array}$ & 0.076 & & & & & & \\
\hline Steep - weak pasterns & & & & & $\begin{array}{c}-0.165 \pm \\
0.131\end{array}$ & 0.208 & & \\
\hline Steep - weak pasterns quad & & & & & $\begin{array}{c}-0.102 \pm \\
0.051\end{array}$ & 0.050 & & \\
\hline
\end{tabular}


${ }^{1}$ Only the characteristics are displayed that retained a $P$-value $<0.1$ in the statistical models after backwards elimination was applied for the elbow joint, femoropatellar joint, tarsocrural joint and animal level. Animal level is the summation of OC scores for the different joints. The total number of animals in the analysis for each model (joint level or animal level) indicated ( $\mathrm{n}=$ ). Due to some missing values, not all 267 animals could be statistically assessed.

${ }^{2}$ The locomotive characteristics were subjectively scored on a scale of 1 to 9 with 1 indicating normal locomotion and higher scores indicating a worse form of locomotion

${ }^{3}$ The conformation characteristics were subjectively scored on a scale of 1 to 9 . Score 5 indicates a normal leg conformation (deviations below or above 5 correspond, respectively, to the conformation characteristic in the name 'conformation name A - conformation name B'), except for the claw size where 5 indicates equally sized claws (deviation below 5 relate to smaller inner claws, deviations above 5 relate to smaller outer claws). Due to these states in the middle 0 the scale, quadratic relationships were investigated. The quadratic terms are signified by 'quad'.

${ }^{4}$ Influence statistics showed high leverage on a few of the observations (see text). After omitting these strong-leverage observations from the analyses, no association was found for these characteristics on OC scores. 
Figure 1. Associations between conformation and locomotive characteristics (CLC) and osteochondrosis (OC) scores. Only the significant associations without leverage abnormalities according to the statistical models between CLC and OC scores are displayed for the elbow joint, femoropatellar joint, tarsocrural joint, and on the animal level. Animal level is the summation of OC scores for the different joints. The Y-axis represents the OC scores from the liability scale as SD units. The X-axes represent the subjectively scored CLC on a scale of 1 to 9. Conformation characteristics are indicated with either an $\mathrm{H}$ or $\mathrm{F}$ in front of the characteristic, indicating that the characteristic was scored in the hind legs or front legs, respectively. Score 5 indicates normal conformation, and scores 1 and 9 indicate severe deviations from normal. Deviations from normal correspond to the name (for example for O shape - X shape, 1 indicates severe $\mathrm{O}$ shaped legs and 9 indicates severe $\mathrm{X}$ shaped legs), except for the claw size where 5 indicates equally sized claws (deviations below 5 relate to smaller inner claws, deviations above 5 relate to smaller outer claws). The locomotive characteristics gait movement / pattern and swaying hindquarters have the optimum value at 1 and the least optimum value at 9. Only the range of scores are visualized for which data were available. The more negative the OC score, the less an animal is affected with OC. 


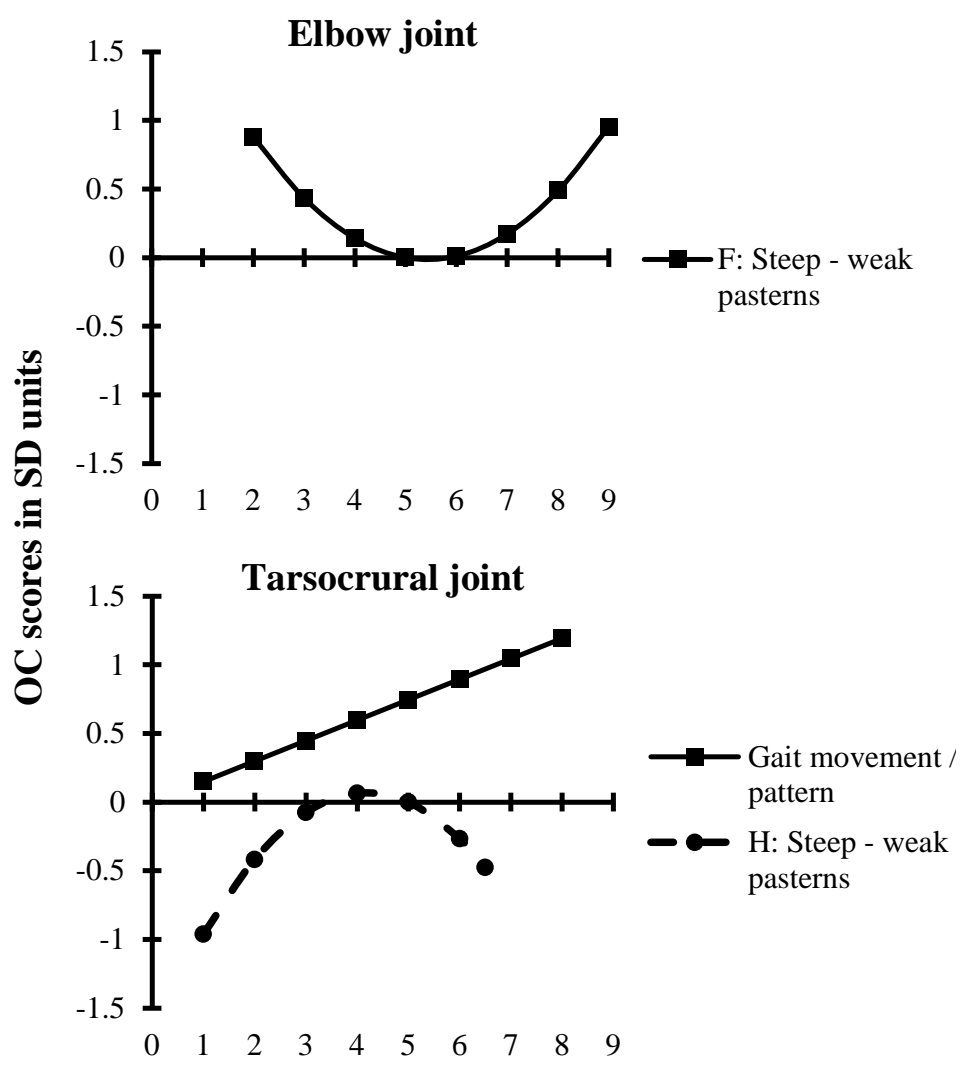

CLC scores
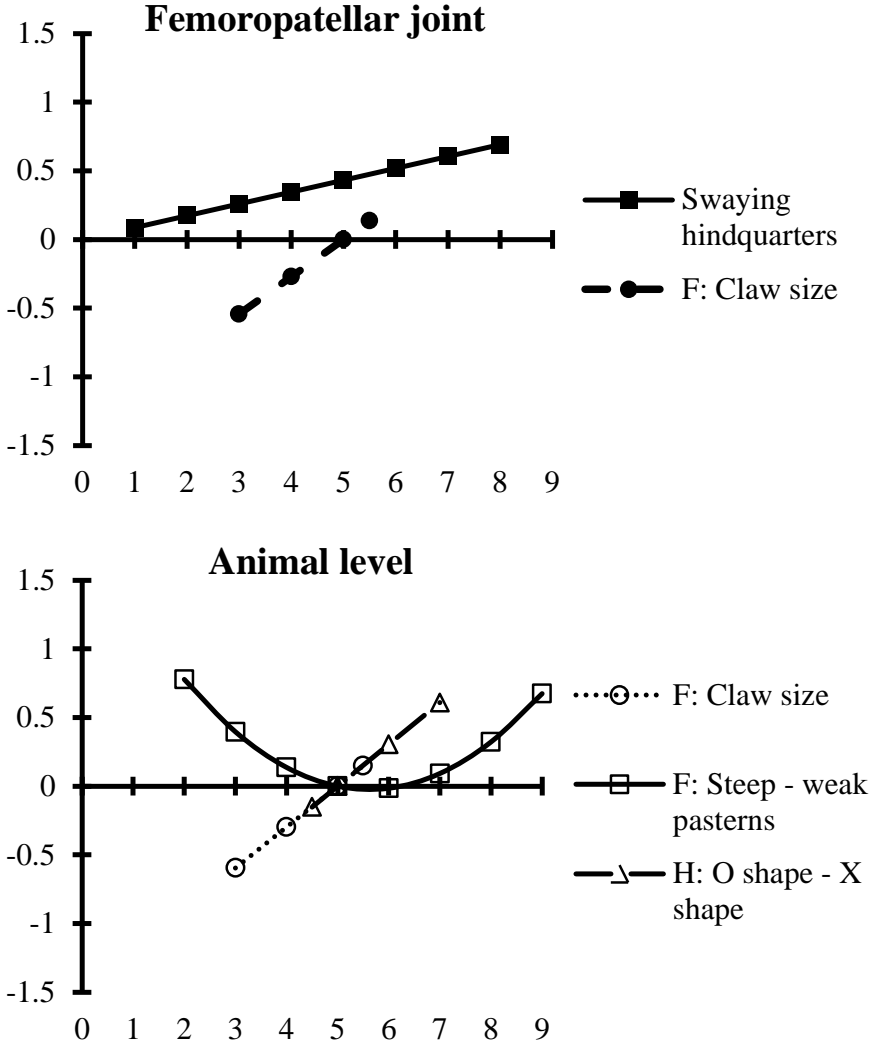

CLC scores 